\title{
Principales remarques du GT SFRP/CIPR concernant le projet de recommandations 2005 de la CIPR
}

\author{
C. SCHIEBER ${ }^{1}$, Y.-S. CORDOLIANI ${ }^{1}$
}

(Manuscrit reçu le 28 décembre 2004, accepté le 10 janvier 2005)

\begin{abstract}
RÉSUMÉ La Commission internationale de protection radiologique a mis l'été dernier sur son site Internet, pour avis, son nouveau projet de recommandations avant de le finaliser courant 2005. Comme pour les ébauches précédentes, la Société française de radioprotection a, au travers d'un groupe de travail, fait ses commentaires sur le projet actuel. Le texte envoyé à la CIPR est présenté ici aux lecteurs de la revue de la SFRP.

ABSTRACT Remarks of the SFRP working group about ICRP recommendations.

The International Commission on Radiological Protection has proposed last summer on its Web site the draft text of the 2005 ICRP recommendations for consultation. As it was done for the previous drafts, the French Society for Radiation Protection, has sent his comments to the ICRP, through a specific working group. The text sent to the ICRP is presented here to the readers of the SFRP's Journal.
\end{abstract}

Key words: CIPR / Recommandations / Radioprotection

\section{Introduction}

Le groupe de travail (GT) créé par la Société française de radioprotection pour étudier les propositions de la CIPR est composé d'une trentaine de membres représentant les parties prenantes de la radioprotection en France : autorités, experts et professionnels des domaines nucléaire, médical et de la recherche, mouvement associatif.

Depuis ses commentaires, soumis à la CIPR début 2004 (Schieber et Cordoliani, 2004), le GT s'est réuni deux fois pour commenter le dernier document proposé par la CIPR (RP05). Étant donné le temps disponible, il n'a pas été possible au GT de rentrer dans le détail des formulations de paragraphes de l'ensemble du document. De façon générale, le GT remarque que ces nouvelles recommandations constituent une évolution du système de radioprotection de la publication 60 de la CIPR (ICRP, 1990), avec une évolution notable de certains

1 Au nom du groupe de travail SFRP. 
concepts, notamment celui de contrainte de dose, qui mériteraient de plus amples explications afin d'améliorer leur compréhension.

Le GT s'est attaché à formuler ses remarques sur les thèmes qui lui paraissaient essentiels. Quatre thèmes principaux ont ainsi été abordés :

- la contrainte de dose,

- le domaine médical,

- l'exclusion,

- les unités et grandeurs dosimétriques.

Ce document présente les principales remarques du GT dans une formulation cherchant à refléter les diverses opinions qui ont pu être exprimées lors des séances de travail.

\section{La contrainte de dose}

Les recommandations 2005 proposent 4 valeurs de contraintes de dose individuelle liées à une source et applicables quelle que soit la situation d'exposition (normale, existante ou d'urgence). Les limites de dose individuelle multi-sources pour les travailleurs et le public sont conservées pour les situations d'exposition normale.

Le GT note que le concept de contrainte de dose devient un outil fort du système de radioprotection. Il apparaît très utile pour la radioprotection du public, où il apporte une certaine flexibilité, notamment pour la gestion des rejets d'installations. Dans le cas de la radioprotection des travailleurs, la majorité des membres du GT estime que cet outil devrait être utilisé principalement par les exploitants, dans le cadre de la démarche d'optimisation, et plus particulièrement pour fixer des contraintes par opération. Une majorité des membres du GT estime qu'il serait souhaitable que les contraintes par source pour l'exposition des travailleurs ne deviennent pas des valeurs réglementaires et infractionnelles.

Dans tous les cas, la définition de «la source » à laquelle est associée une contrainte doit être définie en concertation avec les acteurs concernés.

Le GT note une apparente contradiction entre le $\S 132$ qui spécifie que le respect des contraintes représente un niveau de protection qui devrait être considéré comme obligatoire ( They provide a level of protection that should be considered as obligatory and not maintaining these levels of protection should be regarded as a failure ») et le $\S 137$ qui note que les contraintes pourraient être utilisées comme un outil réglementaire ( Although it may be used as a regulatory tool, so that exceeding a mandatory constraint may be a statutory offence, $\ldots »)$. 
La CIPR pourrait être plus précise sur le caractère réglementaire des contraintes, et notamment sur le sens respectif de «failure » (§ 132) et de « statutory offence » $(\S 137)$.

Dans cet esprit, le GT propose une reformulation du $\S 137: \S 137$ «(..) Although it may be used as a regulatory tool, which in specific circumstances may give rise to a statutory offence when the constraint is exceeded, it will not cause a step change in the associated health risk».

Certains membres du GT estiment que la mention, dans le $\S 164-3^{\circ}$ alinéa, d'une contrainte de dose de $0,3 \mathrm{mSv} / \mathrm{an}$ pour le public en situation normale est trop restrictive et source de confusion étant donné que la valeur de $1 \mathrm{mSv} / \mathrm{an}$ est proposée de façon générique. Si une telle valeur de $0,3 \mathrm{mSv} / \mathrm{an}$ devait être proposée par la CIPR, de plus amples explications devraient être fournies.

\section{Le domaine médical}

Les membres du GT estiment que, de manière générale, des efforts restent à faire pour continuer à renforcer la culture d'optimisation de la radioprotection chez les praticiens de la radiologie. Ils souhaitent que cette nécessité de poursuivre les efforts soit explicitement mentionnée dans le RP05 qui, en l'état actuel, ne donne pas de message suffisamment fort concernant ce sujet.

Pour une majorité des membres du GT, le $\$ 116$ qui mentionne le risque associé à l'exposition d'une grossesse devrait être modifié. Le texte, reprenant pourtant les données des publications 84 et 90 (ICRP, 2000, 2003), dans lesquelles un seuil pour les effets de type malformation était situé «entre 100 et $200 \mathrm{mGy}$ ou supérieur » (Publication 84 de la CIPR (ICRP, 2000) : «malformations have a threshold of 100-200 mGy or higher »), ne propose pas de valeur numérique. Il dit en effet qu'il existe un vrai seuil de 100 mGy d'après les expérimentations animales et que la commission juge qu'on peut écarter le risque de malformation pour des doses de «quelques dizaines de mGy ». Il serait préférable, afin à la fois de permettre la gestion des expositions in utero et d'être cohérent avec les précédentes publications de la CIPR, d'indiquer clairement la valeur de $100 \mathrm{mGy}$ comme seuil pour les malformations humaines.

Le $\$ 116$ devrait donc être réecrit ainsi : «In respect of the induction of malformations, the data strengthen the views that there are gestation agedependant patterns of in utero radiosensitivity with maximum sensitivity being expressed during the period of major organogenesis. Malformations have a threshold of 100-200 mGy or higher; therefore for practical purposes, the Commission judges that risks of malformation after in utero exposure to doses up to 100 mGy may be discounted. » 
En ce qui concerne la justification d'une procédure pour un patient individuel (§ 219), le GT estime qu'il serait utile que la CIPR précise que la justification du choix d'une technique de diagnostic doit prendre en compte la disponibilité du matériel, ainsi que les contraintes économiques et sociales.

Par ailleurs, le $\S 222$ dit que «les procédure médicales entraînant des expositions des patients sont clairement justifiées ». Certains membres considèrent qu'il faudrait ajouter que les procédures sont justifiées « dès lors que les codes de bonnes pratiques ont été établis ».

\section{L'exclusion}

La CIPR recommande des niveaux d'exclusion génériques exprimés en termes de concentration de radionucléides en $\mathrm{Bq} / \mathrm{g}$.

Les valeurs proposées par la CIPR sont contestées par plusieurs membres du GT qui estiment que, dans certaines situations, l'ingestion sur le long terme d'aliments ayant des concentrations de radionucléides égales aux valeurs d'exclusion conduirait à des doses individuelles de plusieurs $\mathrm{mSv}$, ce qui serait inacceptable à leurs yeux.

La formulation du $\S 206$ ( The Commission has concluded that these values provide a practical definition of what is to be considered radioactive... ») laisse à penser que les valeurs d'exclusion définissent la frontière entre ce qui est radioactif et ce qui ne l'est pas, ce qui est également inacceptable pour certains membres du GT et de toute façon physiquement inexact.

Par ailleurs, certains membres du GT estiment que l'on ne doit pas décider de façon générique l'exclusion de matériels radioactifs. Il faut une gestion au cas par cas, avec un contrôle des filières de recyclage. D'autres membres estiment que, sans chercher à banaliser des situations où il y a présence de radioactivité artificielle, il serait utile d'avoir des valeurs d'exclusion notamment pour le démantèlement des installations. Certains membres suggèrent de retenir une valeur en termes de dose (ex. $10 \mu \mathrm{Sv} / \mathrm{an}$ ) et de faire les études au cas par cas, plutôt que d'avoir une approche avec des valeurs génériques en termes de concentration.

\section{Les unités et grandeurs dosimétriques}

Certains membres du GT apprécient la révision des facteurs de pondération pour les neutrons apportée par la CIPR (§68-74) permettant de satisfaire aux lois de la physique tout en ne modifiant pas considérablement la représentativité des grandeurs opérationnelles comme estimateurs de la dose efficace. 
Le GT estime que le remplacement de la dose équivalente par la dose pondérée ( « radiation weighted dose », § 51) est une bonne chose, mais il estime que l'unité $\mathrm{J} \mathrm{kg}^{-1}$ est impropre, et maintient sa proposition d'utiliser le Gray pondéré, noté $\mathrm{D}_{\mathrm{w}}$, ce qui permettrait notamment de ne pas introduire une nouvelle unité en remplacement du Sv de la dose équivalente.

Certains membres du GT souhaiteraient que la CIPR rappelle le domaine de validité des facteurs de pondération permettant de calculer la dose efficace, comme cela était fait dans la publication 26 de la CIPR (ICRP, 1977).

Dans la définition de la dose absorbée (3.3.1), certains membres du GT estiment que la première phrase du $\S 46$ ( $«$ For densely ionising radiation (charged particles from neutrons and alpha particles) and low doses of low LET radiation, the frequency of events in most cells is zero, in a few it is one and extremely exceptionally more than one. ») est ambiguë et mériterait d'être reformulée.

\section{Conclusion}

La Société française de radioprotection remercie la CIPR d'avoir initié une consultation pour le RP05. De façon générale, la majorité des membres du GT apprécie la nouvelle formulation des recommandations et notamment la prise en compte de remarques qui ont pu être exprimées à la CIPR lors des consultations précédentes. Cependant, un effort reste à faire en termes de clarification des concepts et de leur utilisation.

\section{Annexe : Liste des membres du groupe de travail}

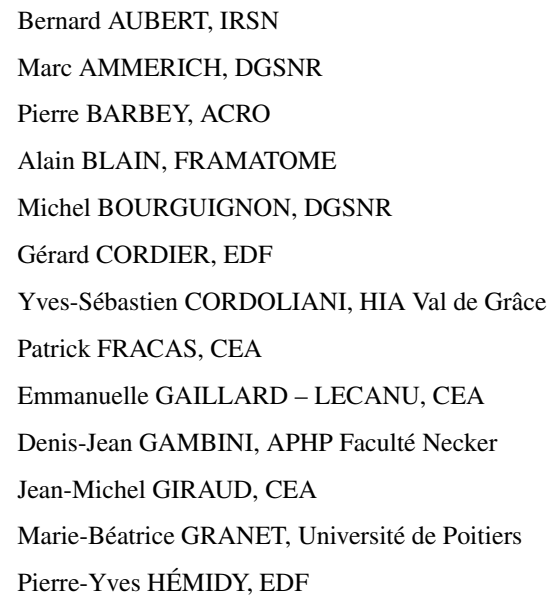

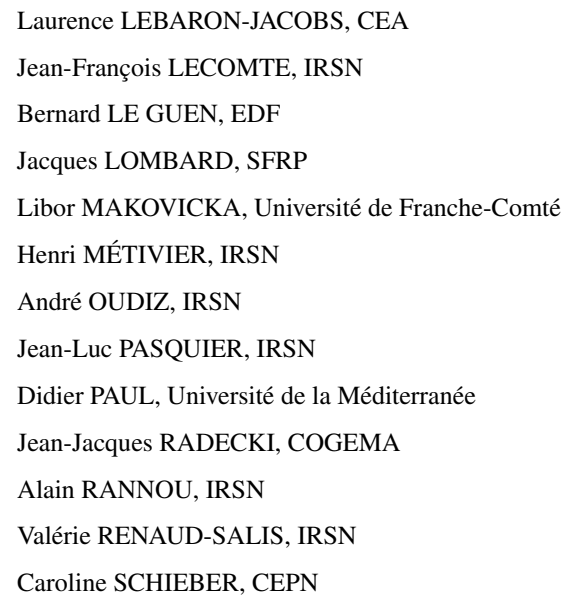




\section{RÉFÉRENCES}

ICRP Publication 26 (1977) Recommendations of the ICRP Annals of the ICRP 1 (3).

ICRP Publication 60 (1990) Recommendation of the International Commission on Radiological Protection, Ann. ICRP 21.

ICRP Publication 84 (2000) Pregnancy and Medical Radiation, Ann. ICRP 30 (1).

ICRP Publication 90 (2003) Biological Effects after Prenatal Irradiation (Embryo and Fetus), Ann. ICRP 33 (1-2).

Schieber C., Cordoliani Y.-S. (2004) Réflexions du groupe de travail de la Société française de radioprotection sur l'évolution du système de radioprotection proposée par la CIPR, Radioprotection 39, 255-267. 